\title{
Dynamic Diffraction Lattice Phase Imaging Using DBI
}

\section{Rodney Herring}

\section{University of Victoria, Victoria, British Columbia, Canada}

A method using diffracted beam interferometry (DBI) [1] to obtain two-dimensional atomic lattice phase images is described showing two examples using crystals of aluminum and gold. These lattice phase images most likely form due to the residual phase contribution of dynamic diffraction within the interfering beams. Dynamic diffraction is complex and the bane of high resolution imaging especially in strongly electron scattering specimens. Being able to measure its contribution to the overall phase image would help to produce better high resolution electron microscopy (HRTEM) lattice images by providing a means for its removal.

The method involves interfering two symmetrical Bragg diffracted beams on the optic axis using an electron biprism (Figure 1a). Kinematically, they should 100\% cancel their phase when interfered on the optic axis to produce a flat phase image. This is not the case. Instead, a residual lattice phase image is produced (Figure 1b,c and Figure 2). The most likely candidate for this residual lattice phase is dynamic diffraction having been picked up by the interfering Bragg diffracted beams during their passage through the crystal specimen.

The phase contributions for a perfect crystal to the interference of the two symmetrically diffracted Bragg beams, ${ }^{\phi} g_{h k l}, \phi_{g_{\bar{h}} \bar{k} \bar{l}}$, on the optic axis is simply given as,

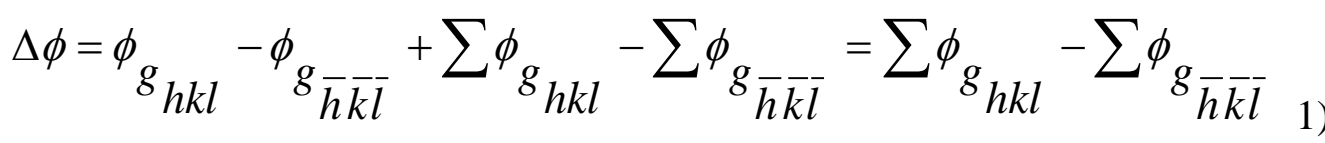

Interestingly, the dynamic lattice phase contributions, $\quad \sum \phi_{g_{h k l}} \neq \sum \phi_{g_{\bar{h} \bar{k} \bar{l}} \text { do not cancel, possibly due }}$ to crystal misalignments, long range crystal strain, point defects or some other phase object(s). Dynamic diffraction has complex contributions to lattice images as there are many possibilities for producing it, especially for strong phase objects like gold and heavy metals.

In this report, aluminum and gold crystals representing weak and strong phase objects, respectively, were used for the two-beam DBI experiments. The residual lattice phase images produced by Al were relatively simple compared to the more complex, residual lattice phase images for Au (Figure 1 and Figure 2).

In these experiments the interference of two symmetrically Bragg diffracted beams on the optic axis revealed residual phase at the crystal's atomic lattice sites producing a lattice phase image. This residual lattice phase measurement offers possibilities to help understand dynamic diffraction's contribution to HREM lattice images with the goal of being able to determine the number and types of atoms contained in the image.

Grants from NSERC Discovery, CFI and BCKDF are greatly appreciated. 

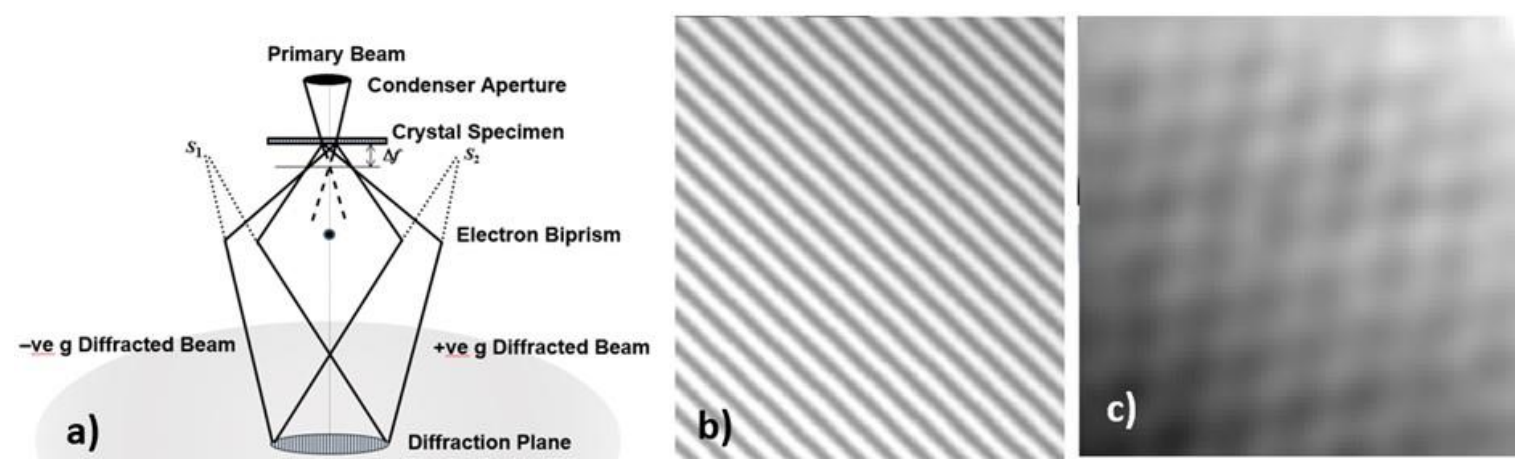

Figure 1. a) Electron optical configuration interfering two symmetrical Bragg diffracting beams on the optic axis by means of an electron biprism, b) DBI interferogram of Al, and c) its reconstructed phase image revealing residual lattice phase.
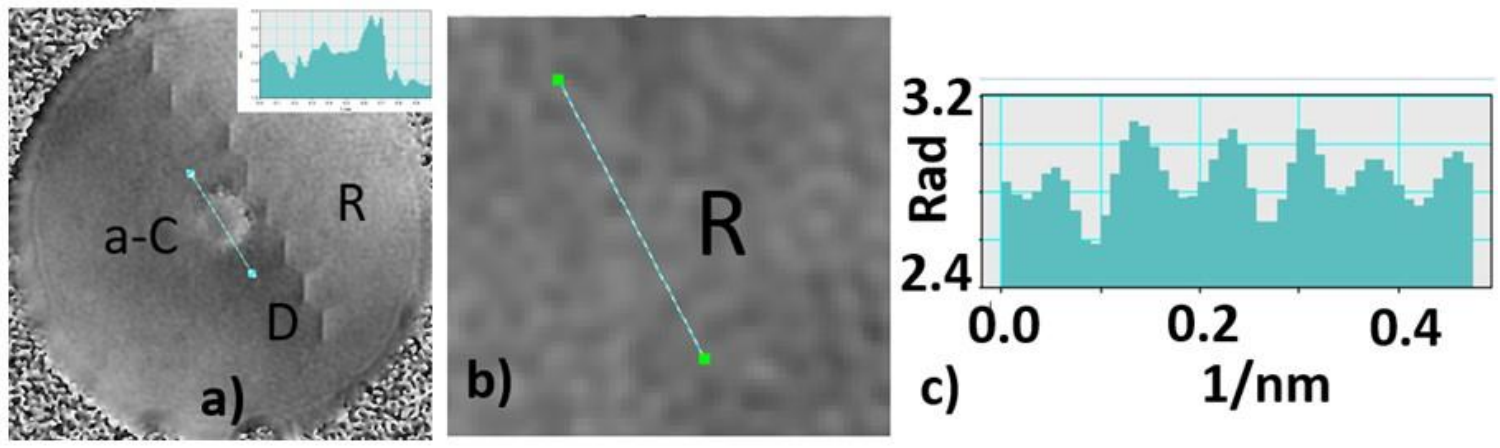

Figure 2. a) DBI phase image of the interference of symmetrical Bragg diffracted beams of Au showing an amorphous $\mathrm{C}$ (a-C) deposit with its phase spectrum passing through the line in the upper right corner,

a dislocation network, D (see [2], [3]), and the residual phase, R, in the perfect crystal magnified in b) and its phase lattice structure along the line shown in c) the phase spectrum clearly revealing the complex phase shift's periodicity.

\section{References}

1. R.A. Herring, G. Pozzi, T. Tanji, A. Tonomura, Ultramicroscopy 60 (1995) 153.

2. R.A. Herring, "Phase Imaging Dislocations using Diffracted Beam Interferometry" Microscopy (2020) MICRO-2020-00047.

3. S. Penkova, R.A. Herring, Microsc. Microanal. 25 (Suppl 2) 2019104. 